\title{
A REMARK ON LOCAL RIGIDITY OF CONFORMAL ACTIONS ON THE SPHERE
}

\author{
MASAHIKo KANAi
}

\section{Introduction}

Let $M$ be a closed orientable $C^{\infty}$ riemannian manifold of dimension $n+1 \geq 3$ with constant sectional curvature -1 . It is covered by the hyperbolic space $H^{n+1}$, and a deck transformation is naturally extended to a $C^{\infty}$ diffeomorphism of the sphere at infinity, which is conformal with respect to the standard metric of the sphere. In consequence, we are provided with a canonical $C^{\infty}$ action of the fundamental group $\Gamma=\pi_{1}(M)$ on the $n$-sphere $S^{n}$, or, alternatively speaking, a homomorphism $\rho_{0}$ of $\Gamma$ into the diffeomorphism group $\operatorname{Diff}\left(S^{n}\right)$. Another action or homomorphism $\rho: \Gamma \rightarrow \operatorname{Diff}\left(S^{n}\right)$ is said to be $C^{k}$-close to $\rho_{0}$, if $\rho(\gamma)$ is close to $\rho_{0}(\gamma)$ relative to the $C^{k}$-topology for finitely many $\gamma \in \Gamma$ that altogether generate $\Gamma$ (recall that $\Gamma$ is finitely generated). One of the main results in the present note is

Theorem A (Rigidity of the action at infinity). $A C^{\infty}$ action $\rho$ of $\Gamma$ which is sufficiently $C^{2}$-close to the standard action $\rho_{0}$ is $C^{\infty}$-conjugate to $\rho_{0}$ (i.e., there exists a $C^{\infty}$ diffeomorphism $h$ of $S^{n}$ such that $\rho(\gamma)=h \circ \rho_{0}(\gamma) \circ h^{-1}$ for all $\gamma \in \Gamma)$.

Let us denote by $\phi_{0}^{t}$ the geodesic flow of $M$ which is defined on the unit tangent bundle $V$ of $M$. It is an Anosov flow with $C^{\infty}$ weak and strong (un)stable foliations. Denote by $\mathcal{F}_{0}^{+}$the weak unstable foliation. We then have

Theorem B (Rigidity of the weak unstable foliation). Any $C^{\infty}$ foliation $\mathcal{F}^{+}$of $V$ of dimension $n+1\left(=\operatorname{dim} \mathcal{F}_{0}^{+}\right)$that is sufficiently close to $\mathcal{F}_{0}^{+}$with respect to the $C^{2}$-topology has to be $C^{\infty}$-conjugate to $\mathcal{F}_{0}^{+}:$Namely, for such a foliation $\mathcal{F}^{+}$, there always exists a $C^{\infty}$ diffeomorphism of $V$ onto itself that sends $\mathcal{F}^{+}$to $\mathcal{F}_{0}^{+}$.

The theorems are false in dimension $n+1=2$, as the hyperbolic structure of $M$ is deformable. There is however a 2-dimensional counterpart due to E. Ghys [G2] (see also $[\mathrm{KY}]$ for an alternative proof). Incidentally, in the succeeding paper [G3], Ghys amazingly established a kind of global rigidity in dimension $n+1=2$.

Received June 29, 1999. Revised October 28, 1999. 
One of the breakthroughs brought by Ghys [G2] is the observation that one can translate a rigidity question for actions of discrete groups into that for foliations. In particular, his method enables us to derive Theorem A from Theorem $\mathrm{B}$ in the following manner (cf. Yue $[\mathrm{Y}]$ ). Given an $C^{\infty}$ action $\rho: \Gamma \rightarrow \operatorname{Diff}\left(S^{n}\right)$, we can form the foliated bundle $\left(M \times{ }_{\rho} S^{n}, \mathcal{H}_{\rho}\right)$ over $M$ : The total space $M \times{ }_{\rho} S^{n}$ is the quotient of $H^{n+1} \times S^{n}$ by the diagonal action of $\Gamma$ (the action on the first component $H^{n+1}$ is by the deck transformations, and on the second via $\rho$ ), while the foliation $\mathcal{H}_{\rho}$ comes from that of $H^{n+1} \times S^{n}$ by the 'horizontal' submanifolds $H^{n+1} \times\{y\}$. This procedure being applied especially to the standard action $\rho_{0}$, the unit tangent bundle $V \rightarrow M$ endowed with the weak unstable foliation $\mathcal{F}_{0}^{+}$ is rebuilt. It can be shown that, if $\rho$ is sufficiently $C^{2}$-close to the standard one $\rho_{0}$, then there exists a $C^{\infty}$ diffeomorphism of $M \times_{\rho} S^{n}$ onto $V$ that maps $\mathcal{H}_{\rho}$ to a foliation which is $C^{2}$-close to $\mathcal{F}_{0}^{+}$. Thus Theorem A follows from Theorem B.

Rigidity theory for actions of lattices of higher-rank semisimple Lie groups has been progressing rapidly: For instance, the 'projective' actions of those groups on some 'boundaries' have recently been shown to be rigid by Katok and Spatzier [KS1], [KS2] (see also [K2] for some partial result). On the contrary, there is no rigidity theorem for actions of rank-one lattices except those by Ghys in dimension $n+1=2$ mentioned earlier. There is however a remarkable attempt by C. Yue, who proved in $[\mathrm{Y}]$ that (a) the standard action $\rho_{0}$ appearing in Theorem A (or, more generally, the actions arising in the same manner from locally symmetric riemannian manifolds of strictly negative curvature) is structurally stable, and that, (b) if the topological conjugacy between the perturbed action and the original one is absolutely continuous, then they are actually conjugate through a $C^{\infty}$ diffeomorphism. One would like to bridge over the gap between these two statements of Yue. This is actually not hard to carry out in the case of real hyperbolic manifolds, as we show here.

\section{Proof of Theorem B}

The first step of the proof of Theorem B is to construct a new Anosov flow $\phi^{t}$ tangent to the perturbed foliation $\mathcal{F}^{+}$, which can be thought of as a perturbation of the geodesic flow $\phi_{0}^{t}$. The new flow $\phi^{t}$ will eventually be shown to be a 'contact' Anosov flow with $C^{\infty}$ strong stable and unstable foliations. Then, the theorem of Benoist, Foulon and Labourie [BFL] would imply Theorem B. We should mention that the stream of our proof is quite similar to that of Yue [Y]. Actually, the present article might be thought of as a small rearrangement of his paper. It should be also pointed out that quite a few of ideas employed in our proof go back to Ghys [G2].

We fix some notation to start with. To denote the strong and weak (un)stable foliations of an Anosov flow, we are going to use the following symbols, attaching " 0 " to them to refer to those that come from the geodesic flow $\phi_{0}^{t}$ : 


\begin{tabular}{|c|c|c|}
\hline & stable foliation & unstable foliation \\
\hline strong & $\mathcal{E}^{-}$ & $\mathcal{E}^{+}$ \\
\hline weak & $\mathcal{F}^{-}$ & $\mathcal{F}^{+}$ \\
\hline
\end{tabular}

Due to the assumption $\mathcal{F}^{+} \approx \mathcal{F}_{0}^{+}$(in $C^{2}$-topology) in Theorem B, we may assume that the tangent bundle $T \mathcal{F}^{+}$of $\mathcal{F}^{+}$is transverse to $T \mathcal{E}_{0}^{-}$, the tangent bundle of the strong stable foliation of the geodesic flow $\phi_{0}^{t}$. There is thus a unique $C^{\infty}$ section $g$ of $T^{*} \mathcal{F}_{0}^{+} \otimes T \mathcal{E}_{0}^{-}$such that

$$
T \mathcal{F}^{+}=\left\{X_{0}^{+}+g\left(X_{0}^{+}\right): X_{0}^{+} \in T \mathcal{F}_{0}^{+}\right\}
$$

that is, to say, $T \mathcal{F}^{+}$is realized as the 'graph' of $g$. Let $Z_{0}$ be the vector field on $V$ that generates the geodesic flow $\phi_{0}^{t}$, and introduce a new $C^{\infty}$ vector field $Z$ of $V$ which is tangent to $\mathcal{F}^{+}$by

$$
Z=Z_{0}+g\left(Z_{0}\right)
$$

The flow generated by $Z$ will be denoted by $\phi^{t}$. It is not hard to see that the diffeomorphisms $\phi^{t}(0 \leq t \leq 1)$ are $C^{2}$-close to $\phi_{0}^{t}$ 's uniformly in $0 \leq t \leq 1$.

Claim 1. $\phi^{t}$ is an Anosov flow which is topologically conjugate to the original one $\phi_{0}^{t}$. Moreover, the strong and weak stable foliations $\mathcal{E}^{-}, \mathcal{F}^{-}$of $\phi^{t}$ coincide with those $\mathcal{E}_{0}^{-}, \mathcal{F}_{0}^{-}$attached to $\phi_{0}^{t}$.

Proof. The first statement is just a standard fact in the theory of Anosov systems. To show that $\mathcal{E}^{-}=\mathcal{E}_{0}^{-}$, recall the following fact: Other than $\mathcal{E}^{-}$, there is no $\phi^{t}$-invariant codimension-one subfoliation of $\mathcal{F}^{-}$that is transverse to the orbits of $\phi^{t}$. Meanwhile, by the definition of the vector field $Z$ that generates $\phi^{t}, \mathcal{E}_{0}^{-}$has to be $\phi^{t}$-invariant. Thus, we are led to $\mathcal{E}^{-}=\mathcal{E}_{0}^{-}$. Finally, to see $\mathcal{F}^{-}=\mathcal{F}_{0}^{-}$, notice that $T \mathcal{F}^{-}$is spanned by $T \mathcal{E}^{-}=T \mathcal{E}_{0}^{-}$and $Z=Z_{0}+g\left(Z_{0}\right)$ with $g\left(Z_{0}\right) \in T \mathcal{E}_{0}^{-}$, while $T \mathcal{F}_{0}^{-}$by $T \mathcal{E}_{0}^{-}$and $Z_{0}$.

Since $\phi^{t}$ is an Anosov flow, there must be constants $\Lambda \geq \lambda>0, c>0$ satisfying the following conditions for all $t \geq 0$ :

$$
\begin{aligned}
c^{-1} \cdot e^{-\Lambda t}\left|X^{-}\right| & \leq\left|d \phi^{t} X^{-}\right| \leq c \cdot e^{-\lambda t}\left|X^{-}\right|, & & X^{-} \in T \mathcal{E}^{-} \\
c^{-1} \cdot e^{\lambda t}\left|X^{+}\right| & \leq\left|d \phi^{t} X^{+}\right| \leq c \cdot e^{\Lambda t}\left|X^{+}\right|, & & X^{+} \in T \mathcal{E}^{+} .
\end{aligned}
$$

Recall that the original flow $\phi_{0}^{t}$ satisfies the same inequalities (in which $T \mathcal{E}^{ \pm}$are to be replaced by $T \mathcal{E}_{0}^{ \pm}$) with $\Lambda=\lambda=c=1$. Since $\phi^{t} \approx \phi_{0}^{t}$ (in $C^{1}$-topology), it follows that the constants $\Lambda, \lambda$ and $c$ for $\phi^{t}$ are all sufficiently close to 1 . In particular, we may assume

$$
1 \leq \Lambda / \lambda<2:
$$

Namely, the '2-pinching condition' on the hyperbolicity of $\phi^{t}$ is fulfilled. The theorem of Hirsch-Pugh [HP] therefore guarantees that the tangent bundles $T \mathcal{E}^{+}$ 
of $\mathcal{E}^{+}$are $C^{1}$-differentiable. Meanwhile, we already know that $T \mathcal{E}^{-}=T \mathcal{E}_{0}^{-}$is $C^{\infty}$.

Let $\theta$ be the 1 -form on $V$ defined by

$$
\theta(Z)=1, \quad \theta(X)=0 \quad\left(X \in T \mathcal{E}^{ \pm}\right) .
$$

It is obvious that $\theta$ is $C^{1}$ and $\phi^{t}$-invariant. Note further that $\theta$ is completely determined by $Z$ and $T \mathcal{E}^{+}$, for $T \mathcal{E}^{-}=T \mathcal{E}_{0}^{-}$is kept invariant under the perturbation of $\mathcal{F}^{+}\left(\right.$or $\left.\phi^{t}\right)$. Due to the assumption, the vector field $Z$ is $C^{1}$-close to $Z_{0}$. In addition, $\phi^{t}(0 \leq t \leq 1)$ is $C^{2}$-close to $\phi_{0}^{t}$. Meanwhile, going back to the proof of the theorem of Hirsch-Pugh [HP], we can easily see that $T \mathcal{E}^{+}$changes $C^{1}$-differentiably when the time-one map $\phi^{1}$ is perturbed in $C^{2}$-topology. It therefore follows that $\theta$ is $C^{1}$-close to $\theta_{0}$, which is by definition the 1 -form defined by $\theta_{0}\left(Z_{0}\right)=1, \theta(X)=0\left(X \in T \mathcal{E}_{0}^{ \pm}\right)$. Since $\theta_{0}$ is the canonical contact form of the unit tangent bundle $V$, we are led to

Claim 2. $\theta$ is a contact form of $V$; that is, $\theta \wedge(d \theta)^{n}$ is a volume form of $V$.

According to Claim 2, $\Omega=\theta \wedge(d \theta)^{n}$ is a continuous volume form of $V$, which is simultaneously $\phi^{t}$-invariant. The regularity theorem of de la Llave-MarcoMoriyón [dLMM] (cf. Hurder-Katok [HK]) guarantees that $\Omega$ is indeed $C^{\infty}$. We are now able to improve the differentiability of $\mathcal{E}^{+}$, appealing to the $\phi^{t}$-invariant smooth volume form $\Omega$. (Incidentally, Yue $[Y]$ proved the following assertion assuming the existence of an invariant volume form $\Omega$, and then derived Claim 2. We go in the opposite way.)

Claim 3. The strong unstable foliation $\mathcal{E}^{+}$of $\phi^{t}$ is also $C^{\infty}$.

Proof. (cf. Ghys [G2], Yue [Y]). Recall that the strong stable foliation $\mathcal{E}^{-}=$ $\mathcal{E}_{0}^{-}$is uniquely ergodic: It has a holonomy-invariant transverse measure that is unique up to constant. Moreover, since $\mathcal{E}^{-}=\mathcal{E}_{0}^{-}$comes from a metric of constant curvature, the invariant transverse measure is represented by a smooth 'transverse volume form' $\tau^{-}$, a nonvanishing $C^{\infty}$ differential form of degree $n+1$ $\left(=\operatorname{codim} \mathcal{E}^{-}\right)$that annihilates the vectors tangent to $\mathcal{E}^{-}$, and is invariant under the holonomy. Since $\mathcal{E}_{0}^{-}=\mathcal{E}^{-}$is invariant not only under $\phi_{0}^{t}$ but also under $\phi^{t}$, the uniqueness of $\tau^{-}$(up to constant) implies $\mathcal{L}_{Z} \tau^{-}=-$const $\cdot \tau^{-}$with const $>0$, where $\mathcal{L}$ denotes the Lie derivative. Define another differential form $\omega^{-}$by $\omega^{-}=i_{Z} \tau^{-}$, with $i$ being the inner product. It also satisfies $\mathcal{L}_{Z} \omega^{-}=-$const $\cdot \omega^{-}$.

Recall that $V$ carries a $\phi^{t}$-invariant $C^{\infty}$ volume form $\Omega=\theta \wedge(d \theta)^{n}$. We can find a unique differential form $\omega^{+}$of degree $n\left(=\operatorname{codim} \mathcal{F}^{+}\right)$that annihilates $T \mathcal{F}^{+}$and satisfies $\omega^{-} \wedge \omega^{+}=i_{Z} \Omega$. Note that $\omega^{+}$is $C^{\infty}$. Take a $C^{\infty} 1$-form $\alpha^{+}$ of $V$ so that it satisfies $d \omega^{+}=\alpha^{+} \wedge \omega^{+}$. We can easily show that the tangent bundle of $\mathcal{E}^{+}$is characterized by $T \mathcal{E}^{+}=\left\{X^{+} \in T \mathcal{F}^{+}: i_{X} \alpha^{+}=0\right\}$ (see [G2], [Y] for a detailed proof). In consequence, $T \mathcal{E}^{+}$turns out to be $C^{\infty}$.

Notice that the claim implies that the contact form $\theta$ is actually $C^{\infty}$.

In the sequel, we have observed that $\phi^{t}$ is an Anosov flow satisfying the following conditions: 
(i) The strong stable and unstable foliations of $\phi^{t}$ are both $C^{\infty}$;

(ii) there is a $\phi^{t}$-invariant $C^{\infty}$ contact form $\theta$ such that $\theta(Z)=1$, where $Z$ is the vector field generating $\phi^{t}$.

We can now apply the theorem of Benoist-Foulon-Labourie [BFL] (see also [G1], $[\mathrm{K} 1],[\mathrm{FK}],[\mathrm{F}]$ for partial results prior to $[\mathrm{BFL}])$ to conclude that $\phi^{t}$ is $C^{\infty}$. conjugate to the original flow $\phi_{0}^{t}$ up to time change. Although the conjugacy between the flows $\phi^{t}$ and $\phi_{0}^{t}$ may not preserve the parameterizations of the flows or the strong (un)stable foliations, it does preserve the weak stable and unstable foliations. It therefore turns out that $\mathcal{F}^{+}$is $C^{\infty}$-conjugate to $\mathcal{F}_{0}^{+}$. This proves Theorem B.

Acknowledgment. The present work was done during the author's visit to École Normale Supérieure de Lyon in 1998. I am grateful to the people working there for their warm hospitality. I also would like to thank Etiennes Ghys for a nice invitation to Lyon and stimulating discussions as well, and to Chengbo Yue for valuable comments.

\section{References}

[BFL] Y. Benoist, P. Foulon, and F. Labourie, Flots d'Anosov à distributions stable et instable différentiables, J. Amer. Math. Soc. 5 (1992), 33-74.

[dLMM] R. de la Llave, R. Marco, and R. Moriyón, Canonical perturbation theory of Anosov systems and regularity results for the Livsic cohomology equation, Ann. of Math. 123 (1986), 537-611.

[F] R. Feres, Geodesic flows on manifolds of negative curvature with smooth horospheric foliations, Ergodic Theory Dynamical Systems 11 (1991), 653-686.

[FK] R. Feres and A. Katok, Anosov flows with smooth foliations and rigidity of geodesic flows on three-dimensional manifolds of negative curvature, Ergodic Theory Dynamical Systems 10 (1990), 657-670.

[G1] E. Ghys, Flots d'Anosov dont les feuilletages satbles sont différentiables, Ann. Sci. École Norm. Sup. (4) 20 (1987), 251-270.

[G2] Déformations de flots d'Anosov et de groupes Fuchsiens, Ann. Inst. Fourier (Grenoble), 42 (1992), 209-247.

[G3] _ Rigidité différentiable des groupes Fuchsiens, Inst. Hautes Études Sci. Publ. Math. 78 (1994), 163-185.

[HP] M.W. Hirsch and C.C. Pugh, Stable manifolds and hyperbolic sets, in Global Analysis, Proc. Sympos. Pure Math., vol.14, pp. 133-163, Amer. Math. Soc., Providence, R.I., 1968.

[HK] S. Hurder and A. Katok, Differentiability, rigidity and Godbillon-Vey classes for Anosov flows, Inst. Hautes Études Sci. Publ. Math. 72 (1990), 5-61.

[K1] M. Kanai, Geodesic flows of negatively curved manifolds with smooth stable and unstable foliations, Ergodic Theory Dynamical Systems 8 (1988), 215-239.

[K2] - A new approach to the rigidity of discrete group actions, Geom. Funct. Anal., 6 (1996), 943-1056.

[KS1] A. Katok and R. Spatzier, Nonstationary normal forms and rigidity of group actions, Electron. Res. Announc. Amer. Math. Soc. 2 (1996), 397-411.

[KS2] Differential rigidity of Anosov actions of higher rank abelian groups and algebraic lattice actions, Tr. Mat. Inst. Steklova 216 (1997), 287-314.

[KY] A. Kononenko and C. Yue, Cohomology and rigidity of Fuchsian groups, Israel J. Math., 97 (1997), 51-59. 
[Y] C. Yue, Smooth rigidity of rank-1 lattice actions on the sphere at infinity, Math. Res. Lett. 2 (1995), 327-338.

Graduate School of Mathematics, Nagoya University, Nagoya 464-8602, JAPAN

E-mail address: kanai@math.nagoya-u.ac.jp 